\title{
Metallicity from Type II supernovae from the (i)PTF ${ }^{\star}$
}

\author{
F. Taddia ${ }^{1}$, P. Moquist ${ }^{1}$, J. Sollerman ${ }^{1}$, A. Rubin ${ }^{2}$, G. Leloudas ${ }^{2,3}$, A. Gal-Yam ${ }^{2}$, I. Arcavi ${ }^{4,5}$, Y. Cao ${ }^{6}$, \\ A. V. Filippenko ${ }^{7}$, M. L. Graham ${ }^{7}$, P. A. Mazzali ${ }^{8,9}$, P. E. Nugent ${ }^{7,10}$, Y.-C. Pan ${ }^{11}$, J. M. Silverman ${ }^{12}$, \\ D. $\mathrm{Xu}^{13}$, and $\mathrm{O}$. Yaron ${ }^{2}$
}

1 The Oskar Klein Centre, Department of Astronomy, Stockholm University, AlbaNova, 10691 Stockholm, Sweden e-mail: ftadd@astro.su.se

2 Department of Particle Physics \& Astrophysics, Weizmann Institute of Science, 76100 Rehovot, Israel

3 Dark Cosmology Centre, Niels Bohr Institute, University of Copenhagen, Juliane Maries Vej 30, 2100 Copenhagen, Denmark

${ }^{4}$ Las Cumbres Observatory Global Telescope, 6740 Cortona Dr, Suite 102, Goleta, CA 93117, USA

5 Kavli Institute for Theoretical Physics, University of California, Santa Barbara, CA 93106, USA

6 Astronomy Department, California Institute of Technology, Pasadena, CA 91125, USA

7 Department of Astronomy, University of California, Berkeley, CA 94720-3411, USA

8 Astrophysics Research Institute, Liverpool John Moores University, 146 Brownlow Hill, Liverpool L3 5RF, UK

9 Max-Planck-Institut für Astrophysik, Karl-Schwarzschild-Str. 1, 85748 Garching, Germany

10 Lawrence Berkeley National Laboratory, Berkeley, CA 94720, USA

11 Astronomy Department, University of Illinois at Urbana-Champaign, 1002 W. Green Street, Urbana, IL 61801, USA

12 Department of Astronomy, University of Texas, Austin, TX 78712, USA

13 Key Laboratory of Space Astronomy and Technology, National Astronomical Observatories, Chinese Academy of Sciences, 20A Datun Road, 100012 Beijing, PR China

Received 16 December 2015 / Accepted 7 February 2016

\section{ABSTRACT}

Type IIP supernovae (SNe IIP) have recently been proposed as metallicity ( $Z$ ) probes. The spectral models of Dessart et al. (2014, MNRAS, 440, 1856) showed that the pseudo-equivalent width of Fe II $\lambda 5018\left(\mathrm{pEW}_{5018}\right)$ during the plateau phase depends on the primordial $Z$, but there was a paucity of SNe IIP exhibiting $\mathrm{pEW}_{5018}$ that were compatible with $Z<0.4 Z_{\odot}$. This lack might be due to some physical property of the SN II population or to the fact that those SNe have been discovered in luminous, metal-rich targeted galaxies. Here we use SN II observations from the untargeted (intermediate) Palomar Transient Factory [(i)PTF] survey, aiming to investigate the $\mathrm{pEW}_{5018}$ distribution of this $\mathrm{SN}$ population and, in particular, to look for the presence of SNe II at lower $Z$. We perform $\mathrm{pEW}_{5018}$ measurements on the spectra of a sample of 39 (i)PTF SNe II, selected to have well-constrained explosion epochs and light-curve properties. Based on the comparison with the $\mathrm{pEW}_{5018}$ spectral models, we subgrouped our $\mathrm{SNe}$ into four $Z$ bins from $Z \approx 0.1 Z_{\odot}$ up to $Z \approx 2 Z_{\odot}$. We also independently investigated the $Z$ of the hosts by using their absolute magnitudes and colors and, in a few cases, using strong-line diagnostics from spectra. We searched for possible correlations between SN observables, such as their peak magnitudes and the $Z$ inferred from $\mathrm{pEW}_{5018}$. We found 11 events with $\mathrm{pEW}_{5018}$ that were small enough to indicate $Z \approx 0.1 Z_{\odot}$. The trend of $\mathrm{pEW}_{5018}$ with $Z$ matches the $Z$ estimates obtained from the host-galaxy photometry, although the significance of the correlation is weak. We also found that $\mathrm{SNe}$ with brighter peak magnitudes have smaller $\mathrm{pEW}_{5018}$ and occur at lower $Z$.

Key words. supernovae: general - Galaxy: abundances

\section{Introduction}

Type II supernovae ( $\mathrm{SNe}$ ) are the most common core-collapse SN events (Li et al. 2011). They are characterized by hydrogenrich spectra (e.g., Filippenko et al. 1997), and their light curves exhibit a fast rise to peak (Rubin et al. 2015, hereafter R15), followed by a long ( $~ 90 \mathrm{~d})$ plateau in the case of SNe IIP or by a linear decline ( $>1.4 \mathrm{mag} / 100 \mathrm{~d})$ in the case of SNe IIL. Anderson et al. (2014) show that these two subclasses may actually be the extremes of a continuum, with several objects showing intermediate light-curve slopes. The nature of the progenitors of SNe IIP is well established: pre-explosion images at their locations show extended ( $R \gtrsim 500 R_{\odot}$ ) red supergiants (RSGs) in the mass range between 8.5 and $17 M_{\odot}$ (Smartt 2009).

Recently, Dessart et al. (2014, hereafter D14) have proposed the use of SNe II as metallicity (Z) probes. In their work, SN II spectral models (first presented in Dessart et al. 2013)

\footnotetext{
* The data are available at the CDS via anonymous ftp to cdsarc.u-strasbg.fr (130.79.128.5) or via http://cdsarc.u-strasbg.fr/viz-bin/qcat?J/A+A/587/L7
}

show that the equivalent width (EW) of metal lines such as Fe II $\lambda \lambda 5018,5169$ depends on the $Z$ of the SN progenitor, as well as on the spectral phase. Also, the pseudo-EW (pEW) of these lines, which is more easily measurable than the actual $\mathrm{EW}$, is a function of $Z$ and phase. D14 measured the pEW of Fe II $\lambda 5018$ [hereafter $\mathrm{pEW}_{5018}$ ] in SN IIP spectra during the plateau phase and compared it with the $\mathrm{pEW}_{5018}$ of their spectral models in order to determine the $Z$ at the $\mathrm{SN}$ locations. Fe II $\lambda 5018$ was chosen because it is easy to observe in SN II spectra and is less affected by line blending than the stronger Fe II $\lambda 5169$ line, whose $\mathrm{pEW}$ is also a proxy for $Z$. Anderson et al. (2015a) recently presented ongoing investigations of the correlation between the $\mathrm{pEW}_{5018}$ and the $\mathrm{SN}$ progenitor $Z$ as measured from the emission lines of $43 \mathrm{SN}$ II host galaxies, at least in the range between $12+\log (\mathrm{O} / \mathrm{H})=8.2-8.6$.

Using spectral data mainly from the Carnegie Supernova Project (CSP), D14 suggest that there is a lack of SNe IIP at $Z \lesssim 0.4 Z_{\odot}$. This could be a characteristic of the SN IIP population, thus providing clues to their progenitor evolution and explosion mechanisms. However, it could also be a bias effect, 
since the CSP mainly observed SNe that were discovered by targeting luminous and therefore metal-rich galaxies. Anderson et al. (2015a) also show a lack of SNe II with small pEW 5018 - that is, at low $Z$ (see their figure 1a). LSQ13fn (Polshaw et al. 2016) shows a small $\mathrm{pEW}_{5018}$, corresponding to $Z \approx 0.1 Z_{\odot}$, but it seems to reside in a solar- $Z$ host galaxy. (On the other hand, it has a large projected offset from the host-galaxy center.) Whether the lack of SNe II at low $Z$ is a bias effect or a property of this SN class can only be tested with a larger sample of events discovered by an untargeted survey.

The Palomar Transient Factory (PTF) and its continuation (the intermediate PTF) are untargeted surveys, which allowed the discovery of a large number of core-collapse $\mathrm{SNe}$ in a wide variety of galaxies. Arcavi et al. (2010) studied the PTF SN populations in dwarf galaxies, finding an excess of $\mathrm{SNe}$ IIb as compared to the SN population in brighter hosts. Thanks to the high cadence of PTF and iPTF [hereafter (i)PTF], for many targets there are also good constraints on the explosion epoch. Furthermore, the (i)PTF collaboration has access to many telescopes for SN follow-up observations (Gal-Yam et al. 2011), and it has collected a large number of high signal-to-noise ratio $(\mathrm{S} / \mathrm{N})$ $\mathrm{SN}$ spectra that are needed to study the pEW of the metal lines.

An extensive sample of (i)PTF SNe II was investigated by $\mathrm{R} 15$ with special focus on their early-time light curves. R15 established the explosion epochs for 57 events, whose spectra show the strong Balmer P-Cygni profiles typical of SNe II. Based on the light curves and the spectra of each SN, we subclassified our SNe into SNe IIP or IIL. Objects with a decline rate $\left(s_{2}\right.$ in Anderson et al. 2014) $>1.4 \mathrm{mag} / 100 \mathrm{~d}$ during the plateau phase and a low ratio between the EW of $\mathrm{H} \alpha$ in absorption and emission were classified as SNe IIL. In Table A.1 we label the SNe IIL with an asterisk "*”. Only five SNe IIL belong to our sample of 39 SNe II.

Here we use the R15 (i)PTF SN sample to investigate the presence of SNe II at low $Z$, by measuring their $\mathrm{pEW}_{5018}$ during the plateau phase. We also check for the correlation between the $Z$ inferred from the $\mathrm{pEW}$ measurements and the values obtained by studying the host-galaxy properties.

This Letter is structured as follows. In Sect. 2 the spectral observations of the (i)PTF SN II sample are presented along with the host-galaxy data. Section 3 describes the EW measurements and the other host-galaxy $Z$ measurements, along with the main results. Our conclusions are summarized in Sect. 4.

\section{Observations}

We collected the optical spectra of the $57 \mathrm{SNe}$ II presented by $\mathrm{R} 15$, as obtained by the (i)PTF collaboration. We looked for $\mathrm{Fe}$ II $\lambda 5018$ in each of the spectra and identified the line in 39 different SNe. For many SNe this line is detected only in a single spectrum, typically the last spectrum obtained during the plateau phase. Even though Fe II $\lambda 2018$ can sometimes be detected before the plateau phase, at those early epochs it is not useful for distinguishing between low and high $Z$ (D14), and that is why only 39 out of $57 \mathrm{SNe}$ were analyzed. For the SNe where the line was detected at multiple epochs during the plateau phase, we selected the spectrum with the highest $\mathrm{S} / \mathrm{N}$ for further analysis.

The selected spectra were obtained with many different telescopes and instruments, as summarized in Table A.1. Each spectrum has been reduced in the standard manner, including bias and flat-field corrections, wavelength calibration using the spectrum of a comparison lamp, and flux calibration with the spectrum of a standard star observed on the same night.
For each spectrum where Fe II $\lambda 5018$ was identified, we established the phase, based on the explosion date reported by R15. The phase was corrected for time dilation based on the SN redshift (from R15), even though this correction is minimal for our relatively nearby objects. (The average redshift of our sample is $\bar{z}=0.030$.) The phase was determined with high accuracy $( \pm 1.15 \mathrm{~d}$ on average) given the high cadence of (i)PTF.

We also collected photometry (ugriz) and optical spectra of the host galaxies of our SNe from the Sloan Digital Sky Survey (SDSS; Ahn et al. 2014). Of our 39 SNe, 35 are in the SDSS footprint and have a detected host. An SDSS spectrum is available for only 14 of our galaxies. Using these data we are able to independently check the $Z$ estimates from $\mathrm{pEW}_{5018}$.

\section{Analysis and results}

We measured $\mathrm{pEW}_{5018}$ with a MATLAB script based on the formulae given by Nordin et al. (2011; see their Eqs. (1) and (2)). The uncertainty estimates include the error due to the pseudocontinuum selection and that associated with the noise of the spectrum. The boundaries of the continuum were selected manually with the help of a smoothed spectrum on top of the original data to guide the eye. We compared these EW measurements and uncertainty estimates with those obtained with the IRAF splot EW tool and found that the results were consistent.

We plot $\mathrm{pEW}_{5018}$ as a function of SN phase in Fig. 1, also showing the models by D14 for different $Z$. We indicate $\mathrm{pEW}_{5018}$ measurements from D14, mainly from CSP SNe II. Also, the $\mathrm{pEW}_{5018}$ values at $+50 \mathrm{~d}$ presented by Anderson et al. (2015a) are provided. Between $\sim 60$ and $\sim 90 \mathrm{~d}$, the $\mathrm{pEW}_{5018}$ values inferred for the objects in our sample are on average lower than what was previously presented in the literature. The untargeted nature of the (i)PTF survey, along with its spectroscopic followup capability, has allowed us to find a dozen SNe II (black symbols in Fig. 1) whose $\mathrm{pEW}_{5018}$ match spectral models having $Z=0.1 Z_{\odot}$ (black line in Fig. 1). In some cases these $\mathrm{SNe}$ have even smaller $\mathrm{pEW}_{5018}$ than what is expected from these models. Only LSQ13fn (Polshaw et al. 2016) has a comparably small $\mathrm{pEW}_{5018}$ (see the magenta empty circle in Fig. 1). In Fig. 2 we show a few examples of (i)PTF SN II spectra selected among those with small $\mathrm{pEW}_{5018}$. This line is particularly faint, but clearly detected given the high $\mathrm{S} / \mathrm{N}$ of these spectra.

Because of the small number of available host-galaxy spectra, we resorted to using the photometric measurements of the SN host galaxies from SDSS to test whether the SNe with small $\mathrm{pEW}_{5018}$ are indeed in small metal-poor galaxies, and if those with large $\mathrm{pEW}_{5018}$ are in large, luminous, metal-rich galaxies. First, we converted the $r$-band apparent magnitudes from SDSS (Cmodel) to absolute magnitudes $\left(M_{\text {host }}(r)\right)$ using the distance moduli presented by R15 and $E(B-V)_{\text {MW }}$ from Schlafly \& Finkbeiner (2011). Figure A.1 shows $\mathrm{pEW}_{5018}$ versus $M_{\text {host }}(r)$ (excluding SNe IIL). Even if the phases of the spectra span at least two months, there is a correlation between the two observables (Spearman test gives $p$-value $=0.007$ ). In our sample, SNe with $\mathrm{pEW}_{5018} \lesssim-20 \AA$ never occur in galaxies fainter than $M_{\text {host }}(r) \approx-19 \mathrm{mag}$. Then, using $M_{\text {host }}(r)$, we obtained an estimate of the metal content $\left(Z_{\text {host }}\right)$ for each host via Eq. (1) of Arcavi et al. (2010). We plot in Fig. 3 (top-left panel) the cumulative distributions of $Z_{\text {host }}$ for the host galaxies of the $\mathrm{SNe}$ with $\mathrm{pEW}_{5018}$ consistent with $Z_{\mathrm{SN}} \approx 0.1,0.4,1$, and $2 Z_{\odot}$.

We subdivided our SNe into these four $Z$ bins based on the distance of their $\mathrm{pEW}_{5018}$ values from those of the models by D14 (see Fig. 1). It indeed seems that $\mathrm{SNe}$ with the largest $\mathrm{pEW}_{5018}$ at a given phase are in galaxies with the 


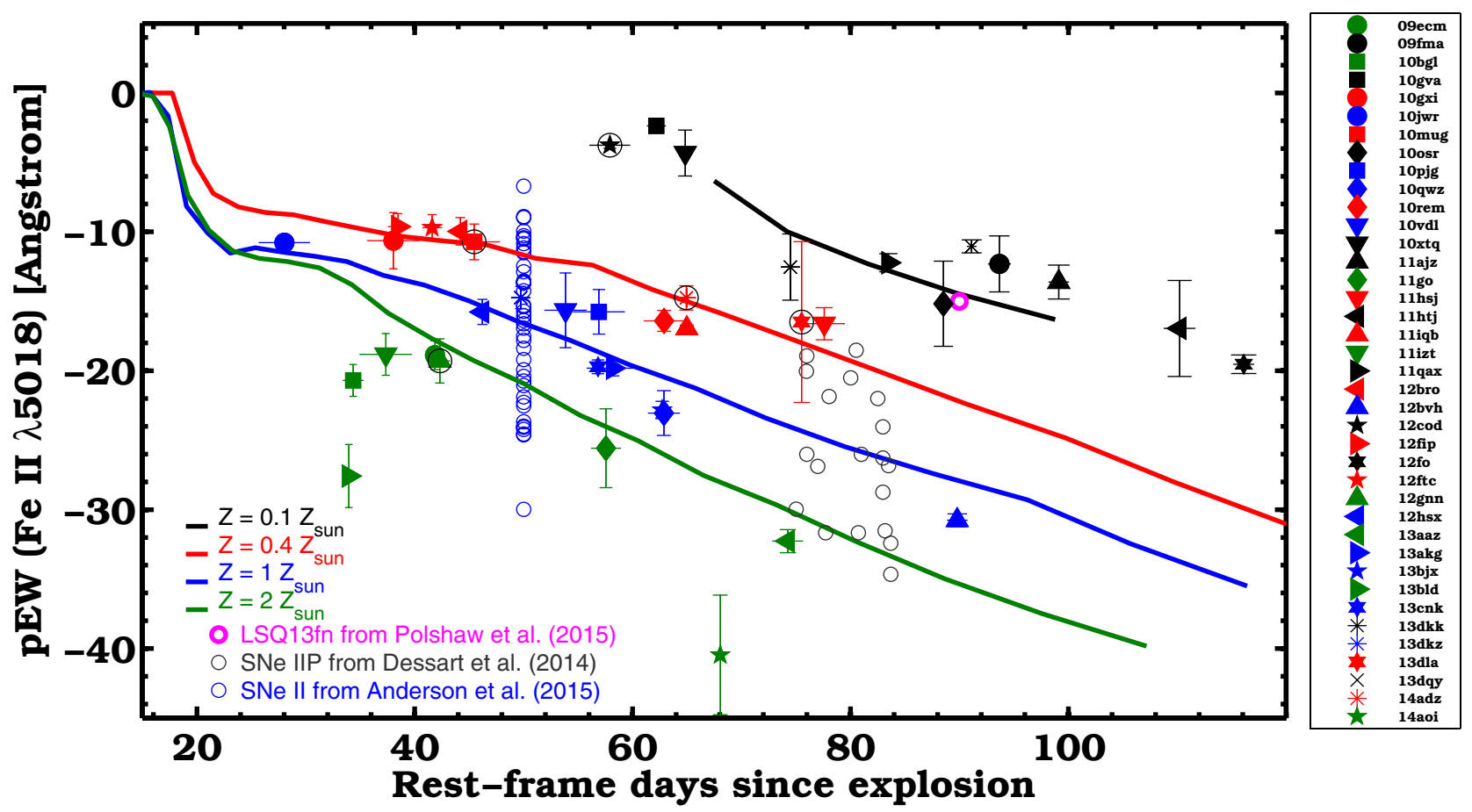

Fig. 1. $\mathrm{pEW}_{5018}$ as a function of the SN phase. Filled symbols label our (i)PTF SNe II. Most of them are SNe IIP, and those circled in black are SNe IIL (i.e., they show a decline rate $>0.014$ mag d $^{-1}$ on the plateau). The spectral models by D14 indicating different $Z$ are represented by solid lines. Our SNe are subgrouped and color-coded in 4 subsets based on the $\mathrm{pEW}(\mathrm{t})$ model to which they appear closest. pEW measurements from D14 and Anderson et al. (2015a) are shown by empty circles (gray and blue, respectively). Our SN sample includes a subset of objects with unprecedentedly small $\mathrm{pEW}$, consistent with $Z_{\mathrm{SN}}=0.1 Z_{\odot}$. The only object with similar pEW(Fe II 5018) is LSQ13fn (Polshaw et al. 2016).

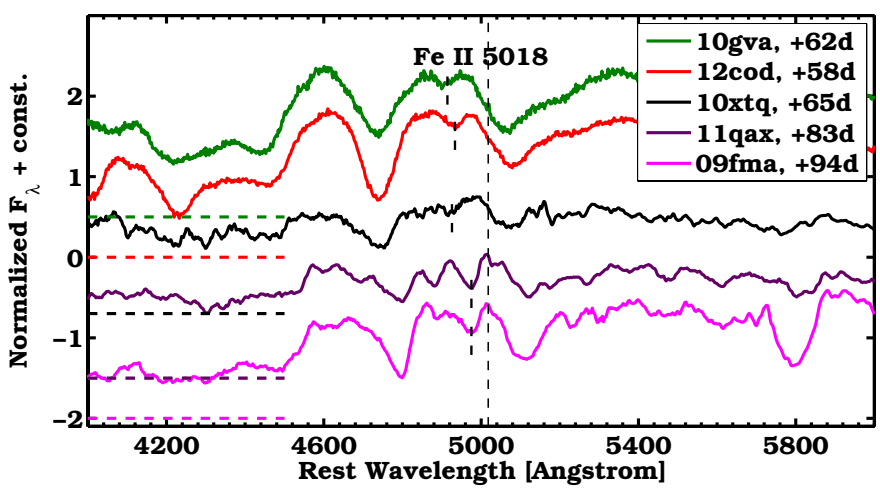

Fig. 2. Examples of SN II spectra with small pEW 5018 . The Fe II $\lambda 5018$ rest wavelength is marked by a vertical dashed line, the absorption minima by vertical dashed segments. The spectra are normalized by their median, offset by a constant (dashed horizontal lines), and shown in the rest frame.

highest amounts of metals (see green line). Since the luminositymetallicity (LZ) relation from Arcavi et al. (2010, see also Tremonti et al. 2004) is known to be affected by large dispersion, we also estimate the host $Z$ via the luminosity-color-metallicity (LCZ) relation by Sanders et al. (2013). Making use of their O3N2 calibration along with $M_{\text {host }}(g)$ and $(g-r)_{\text {host }}$ for each host in order to get the oxygen abundances, these abundances were then converted into $Z_{\text {host }}$. In the top-right panel of Fig. 3, we show that with this improved calibration the SNe with smaller $\mathrm{pEW}_{5018}$ (black and red lines) are also located in metal-poorer galaxies.

To estimate the $Z$ at the location of our SNe within their hosts, we can correct the global $Z$ of their hosts for the metallicity gradient that is known to characterize galaxies (e.g., Pilyugin et al. 2004; Taddia et al. 2013, 2015), where the nucleus is
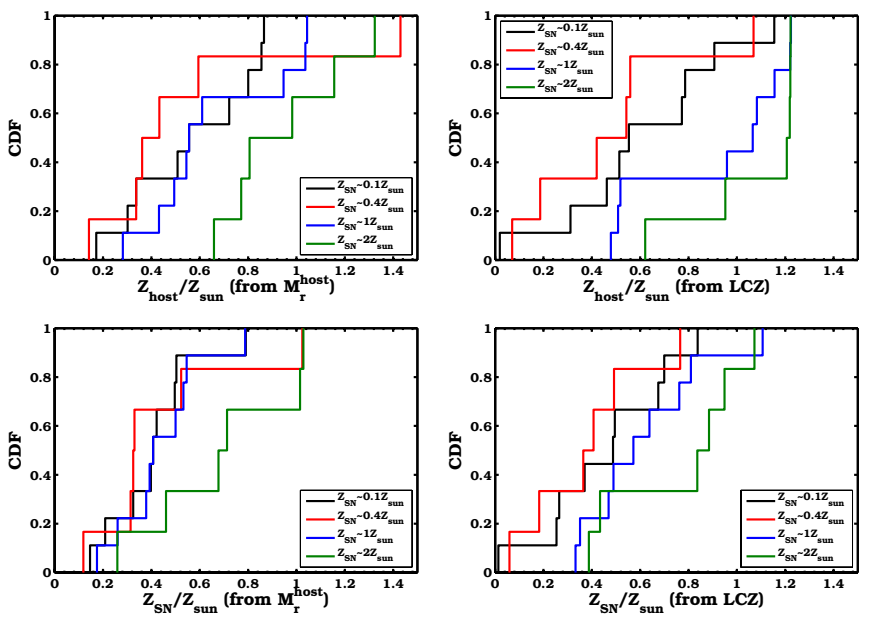

Fig. 3. Top panels: global metallicity $\left(Z^{\text {host }}\right)$ cumulative distributions from the LZ (left) and the LCZ (right) relations. We subdivided the SNe IIP into $4 Z$ bins based on their $\mathrm{pEW}_{5018}$ as compared to the models by D14. Bottom panels: as in the top panels, but $Z$ is that at the $\mathrm{SN}$ locations, as derived by assuming a (single) $Z$ gradient for all the hosts.

typically more metal-rich than the outer parts. We used the derived global $Z$ as a proxy of the central $Z$, and then adopted an average $Z$ gradient of $-0.47 R_{25}^{-1}$ (see Pilyugin et al. 2004). The deprojected and radius-normalized distance for each SN from its host center (see Table A.2) was estimated using the $\mathrm{SN}$ and the host-galaxy coordinates, the host-galaxy radius, the host-galaxy axis ratio, and the position angle as obtained from SDSS (Ahn et al. 2014). In Fig. 3 we show the obtained SN location cumulative $Z$ distributions for the four SN groups based on $\mathrm{pEW}_{5018}$, using the $\mathrm{LZ}$ relation (bottom-left panel) and the LCZ relation (bottom-right panel). With the LCZ calibration, 


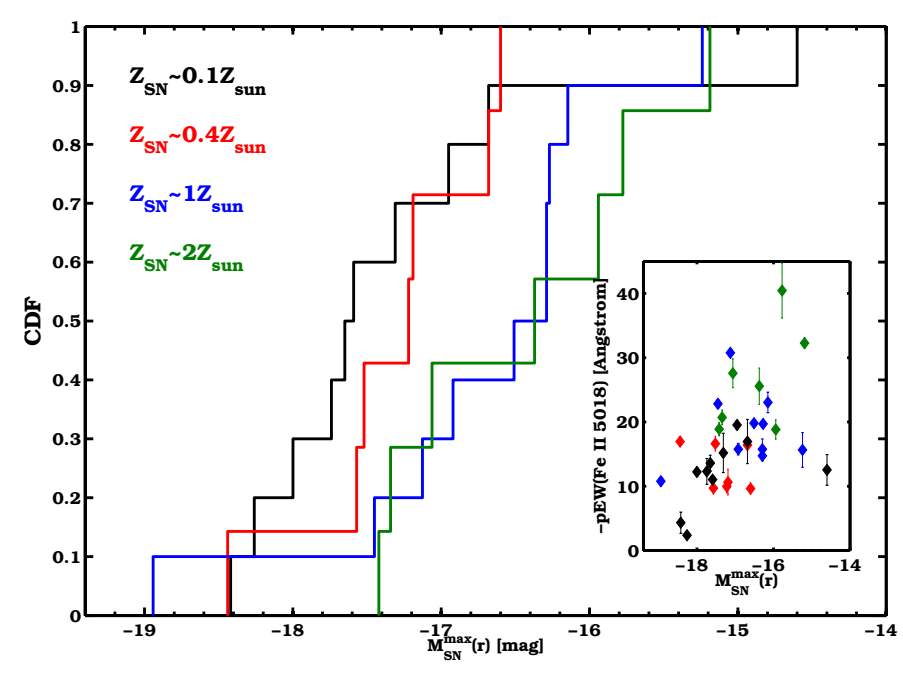

Fig. 4. Cumulative distributions of the $\mathrm{SN}$ peak $r$-band absolute magnitude for the four $Z$ bins based on $\mathrm{pEW}_{5018}$. SNe IIP at lower $Z$ tend to be more luminous.

$\mathrm{pEW}_{5018}$ is confirmed as a proxy for the actual SN host $Z$, with the objects having smaller $\mathrm{pEW}_{5018}$ located at lower $Z$. In all the distributions of Fig. 3, we only included SNe IIP, but including $\mathrm{SNe}$ IIL does not change the results significantly. Spearman tests between $Z_{\mathrm{SN}}$ from $\mathrm{pEW} \mathrm{W}_{5018}$ and $Z_{\text {host }}$ (from both LC and LCZ) reveal that there is a correlation with $p$-values $<0.05$. All the $Z$ estimates are reported in Table A.2.

The values of $Z$ from $\mathrm{pEW}_{5018}$ cover a wider range than those from LZ and LCZ (see Fig. 3, and also Anderson et al. 2016, their Fig. 10). The latter are obtained from $O$ abundances, whereas $\mathrm{pEW}_{5018}$ is essentially a measure of the $\mathrm{Fe}$ abundance. The D14 models assume a constant $[\mathrm{O} / \mathrm{Fe}]$, but at least in the MW $[\mathrm{O} / \mathrm{Fe}]$ is lower at higher $[\mathrm{Fe} / \mathrm{H}]$ (e.g., Amarsi et al. 2015). Therefore, $\mathrm{SNe}$ with low $\mathrm{pEW}_{5018}$ will be found at higher hostgalaxy $Z$ (based on $\mathrm{O}$ abundance) than expected from the models based on $\mathrm{Fe}$ abundance, and vice versa.

In Table A.2, we also report the $Z$ measurements from the emission lines of the few SDSS galaxy spectra that are available and whose line ratios were consistent with no AGN contamination (Baldwin et al. 1981).

We tested if the different $\mathrm{SN}$ groups based on $\mathrm{pEW}_{5018}$ have different $\mathrm{SN}$ observables. The K-S tests show that there is no statistically significant difference among the four groups when we compare the distributions of $r$-band rise time and $r$-band $\Delta m_{15}$. (SN properties were taken from R15.) However, we found that there is a statistically significant difference between the low- and high-Z SN groups when we compare their absolute $r$-band peak magnitudes $\left[M_{\mathrm{SN}}^{\max }(r)\right]$. These were corrected for the host extinction by measuring the EW of the narrow Na I D (Turatto et al. 2003). SNe at lower $Z\left(Z \approx 0.1 ; 0.4 Z_{\odot}\right)$ tend to be more luminous than those at high $Z\left(Z \approx 1 ; 2 Z_{\odot}\right)$, with only a $1 \%$ chance of being drawn from the same distribution. The $M_{\mathrm{SN}}^{\max }(r)$ distributions are shown in Fig. 4. The average peak magnitudes of low- and high-Z SNe are $\left\langle M_{\mathrm{SN}}^{\max }\right\rangle=-17.3 \mathrm{mag}$ and $-16.6 \mathrm{mag}$, respectively. In the inset of Fig. 4 , we also show that $\mathrm{pEW}_{5018}$ measured at different phases during the plateau correlates with the SN peak magnitude. Models of SN II progenitors with initial mass $=15 M_{\odot}$ and different $Z$ by Dessart et al. (2013) show that the $V$-band peak should be fainter for low- $Z$ SNe because they explode with more compact radii, in contrast to our trend. However, their $M_{\mathrm{SN}}^{\max }(r)$ range is narrower than 1 mag, whereas our observed $\mathrm{SNe}$ span 4 mag.

\section{Conclusions}

SNe IIP were known to occur at relatively high $Z$ (Anderson et al. 2010; D14). Thanks to the untargeted (i)PTF survey, we have shown that SNe IIP also arise in relatively large numbers from progenitors consistent with $Z \approx 0.1 Z_{\odot}$. The high quality of the (i)PTF spectra allows us to also measure the weakest Fe II $\lambda 5018$ lines. The expected trend in $\mathrm{pEW}(t)$ with $Z_{\text {host }}$ is observed, although with weak significance. SNe IIP with smaller $\mathrm{pEW}$ tend to occur in metal-poorer environments. Spectral $Z$ measurements are required to better calibrate the relation and assess its dispersion (see, e.g., Anderson et al. 2015a). SN IIP peak magnitudes correlate with $Z$, with more-luminous SNe occurring at lower $Z$.

Acknowledgements. We gratefully acknowledge the support from the Knut and Alice Wallenberg Foundation. The Oskar Klein Centre is funded by the Swedish Research Council. A.G.-Y. is supported by the EU/FP7 via ERC grant No. 307260, the Quantum Universe I-Core program by the Israeli Committee for Planning and Budgeting and the ISF; by Minerva and ISF grants; by the Weizmann-UK "making connections" program; and by Kimmel and ARCHES awards. A.V.F.'s research is supported by the Christopher R. Redlich Fund, the TABASGO Foundation, and NSF grant AST-1211916. We are grateful to the staffs at the many observatories where data for this study were collected (Palomar, Lick, Keck, etc.). We thank R. Foley, J. Bloom, Green, N. Cucchiara, A. Horesh, K. I. Clubb, M. T. Kandrashoff, K. Maguire, A. De Cia, S. Tang, B. Zackay, B. Sesar, A. Waszczak, I. Shivvers, who helped with some of the observations and data reduction. Research at Lick Observatory is partially supported by a generous gift from Google. Some of the data presented herein were obtained at the W. M. Keck Observatory, which is operated as a scientific partnership among the California Institute of Technology, the University of California, and NASA; the observatory was made possible by the generous financial support of the W. M. Keck Foundation. This research used resources of the National Energy Research Scientific Computing Center, a DOE Office of Science User Facility supported by the Office of Science of the US Department of Energy under Contract No. DE-AC02-05CH11231. J.M.S. is supported by an NSF Astronomy and Astrophysics Postdoctoral Fellowship under award AST-1302771. D.X. acknowledges the support of the One-Hundred-Talent Program from the National Astronomical Observatories, Chinese Academy of Sciences. This work is partly based on the Bachelor thesis by P. Moquist.

\section{References}

Ahn, C. P., Alexandroff, R., Allende Prieto, C., et al. 2014, ApJS, 211, 17 Amarsi, A. M., Asplund, M., Collet, R., \& Leenaarts, J. 2015, MNRAS, 454, L11

Anderson, J. P., Covarrubias, R. A., James, P. A., Hamuy, M., \& Habergham, S. M. 2010, MNRAS, 407, 2660

Anderson, J. P., González-Gaitán, S., Hamuy, M., et al. 2014, ApJ, 786, 67

Anderson, J. P., Gutiérrez, C. P., \& Dessart, L. 2015, arXiv e-prints [arXiv: 1510.04271$]$

Anderson, J. P., Gutierrez, C. P., Dessart, L., et al. 2016, A\&A, accepted [arXiv: 1602.00011]

Arcavi, I., Gal-Yam, A., Kasliwal, M. M., et al. 2010, ApJ, 721, 777

Baldwin, J. A., Phillips, M. M., \& Terlevich, R. 1981, PASP, 93, 5 (BPT)

Dessart, L., Hillier, D. J., Waldman, R., \& Livne, E. 2013, MNRAS, 433, 1745

Dessart, L., Gutierrez, C. P., Hamuy, M., et al. 2014, MNRAS, 440, 1856 [D14] Filippenko, A. V. 1997, ARAA, 35, 309

Gal-Yam, A., Kasliwal, M. M., Arcavi, I., et al. 2011, ApJ, 736, 159

Li, W., Chornock, R., Leaman, J., et al. 2011, MNRAS, 412, 1473

Nordin, J., Östman, L., Goobar, A., et al. 2011, A\&A, 526, A119

Pettini, M., \& Pagel, B. E. J. 2004, MNRAS, 348, L59

Pilyugin, L. S., Vílchez, J. M., \& Contini, T. 2004, A\&A, 425, 849

Polshaw, J., Kotak, R., Dessart, L., et al. 2016, A\&A, in press, DOI: $10.1051 / 0004-6361 / 201527682$

Rubin, A., Gal-Yam, A., De Cia, A., et al. 2015, ApJ, submitted [arXiv: 1512.00733] [R15]

Sanders, N. E., Levesque, E. M., \& Soderberg, A. M. 2013, ApJ, 775, 125

Schlafly, E. F., \& Finkbeiner, D. P. 2011, ApJ, 737, 103

Smartt, S. J. 2009, ARA\&A, 47, 63

Taddia, F., Sollerman, J., Razza, A., et al. 2013, A\&A, 558, A143

Taddia, F., Sollerman, J., Fremling, C., et al. 2015, A\&A, 580, A131

Tremonti, C. A., Heckman, T. M., Kauffmann, G., et al. 2004, ApJ, 613, 898

Turatto, M., Benetti, S., \& Cappellaro, E. 2003, From Twilight to Highlight: The

Physics of Supernovae, 200 
F. Taddia et al.: Metallicity from Type II supernovae from the (i)PTF

Appendix A: Additional figure and tables

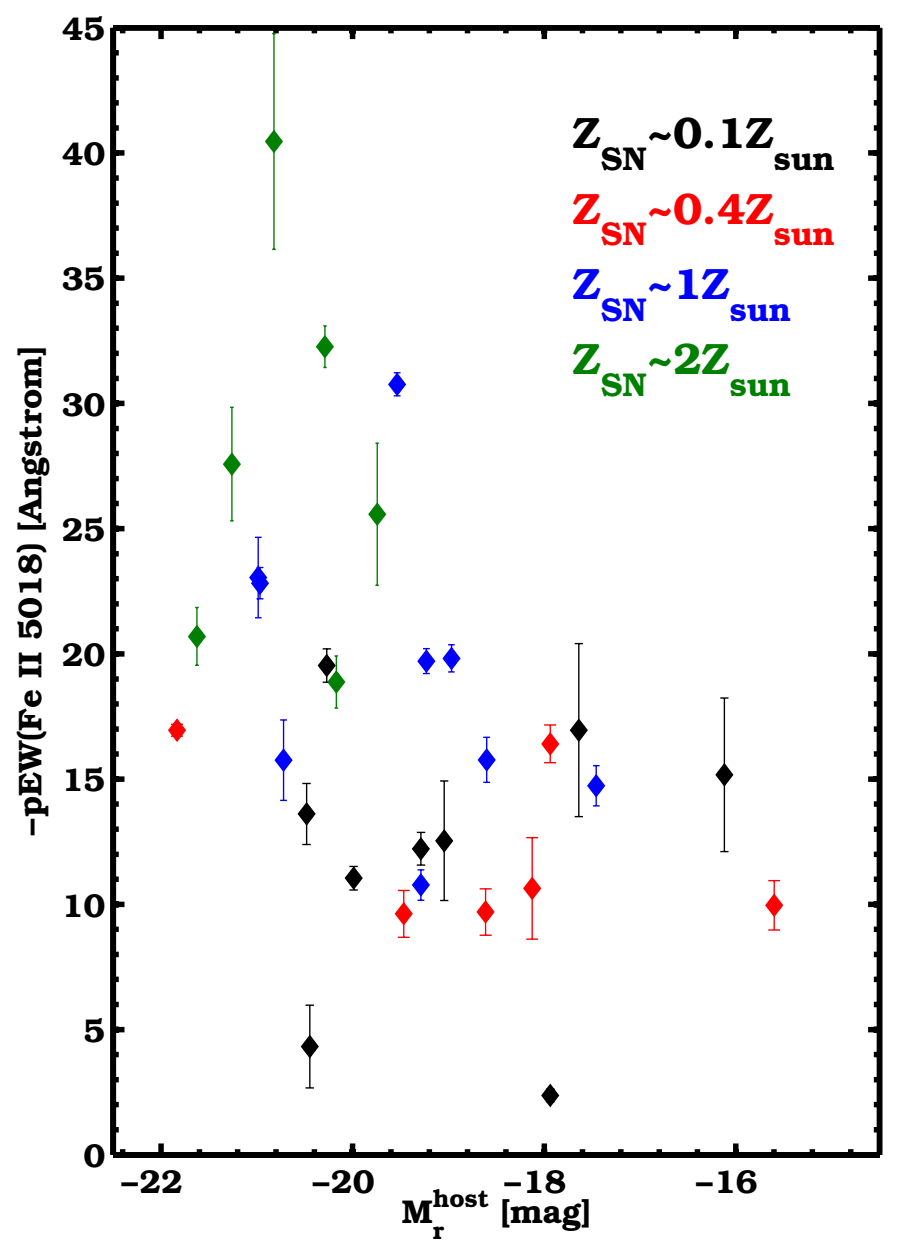

Fig. A.1. $\mathrm{pEW}_{5018}$ versus $M_{r}^{\text {host }}$. Symbols are color-coded as in Fig. 1.
Table A.1. Log of spectral observations and pEW measurements.

\begin{tabular}{|c|c|c|c|c|}
\hline (i)PTF SN & $\begin{array}{l}\text { Phase }^{a} \\
\text { (d) }\end{array}$ & $\begin{array}{l}-\mathrm{pEW}_{5018} \\
(\AA)\end{array}$ & Telescope+Instrument & $\begin{array}{l}\text { Date } \\
\text { (UT) }\end{array}$ \\
\hline$\overline{09 e c m}$ & $41.87(0.35)$ & $18.88(1.04)$ & Keck1+LRIS & 2009 Oct. 23 \\
\hline 09fma & $93.66(1.05)$ & $12.31(2.01)$ & P200+DBSP & 2010 Jan. 09 \\
\hline 10bgl & $34.35(0.97)$ & $20.70(1.15)$ & Keck1+LRIS & 2010 Feb. 06 \\
\hline 10gva & $62.17(0.88)$ & $2.37(0.26)$ & Keck1+LRIS & 2010 Jun. 12 \\
\hline $10 \mathrm{gxi}$ & $38.04(2.41)$ & $10.64(2.02)$ & P200+DBSP & 2010 Jun. 13 \\
\hline 10jwr & $28.03(2.35)$ & $10.77(0.60)$ & Keck1+LRIS & 2010 Jul. 07 \\
\hline $10 \mathrm{mug}^{*}$ & $45.46(2.35)$ & $10.72(1.28)$ & P200+DBSP & 2010 Aug. 14 \\
\hline $10 \mathrm{osr}$ & $88.53(0.91)$ & $15.17(3.07)$ & Lick 3-m+Kast & 2010 Oct. 11 \\
\hline 10pjg & $56.91(2.39)$ & $15.75(1.60)$ & P200+DBSP & 2010 Sep. 06 \\
\hline $10 \mathrm{qwz}$ & $62.87(1.47)$ & $23.04(1.61)$ & Lick 3-m+Kast & 2010 Oct. 11 \\
\hline 10rem & $62.89(1.87)$ & $16.41(0.75)$ & Keck2+DEIMOS & 2010 Oct. 12 \\
\hline $10 \mathrm{vdl}$ & $53.84(1.95)$ & $15.65(2.69)$ & Keck2+DEIMOS & 2010 Nov. 07 \\
\hline $10 x t q$ & $64.82(0.45)$ & $4.32(1.65)$ & P200+DBSP & 2010 Dec. 06 \\
\hline 11ajz & $99.12(0.94)$ & $13.61(1.22)$ & Lick 3-m+Kast & 2011 May 13 \\
\hline 11go & $57.55(1.37)$ & $25.57(2.84)$ & P200+DBSP & 2011 Mar. 10 \\
\hline $11 \mathrm{hsj}$ & $77.60(1.90)$ & $16.61(1.16)$ & Lick 3-m+Kast & 2011 Sep. 29 \\
\hline $11 \mathrm{htj}$ & $110.21(1.46)$ & $16.96(3.45)$ & P200+DBSP & 2011 Oct. 30 \\
\hline $11 \mathrm{iqb}$ & $64.97(0.21)$ & $16.95(0.24)$ & Keck1+LRIS & 2011 Sep. 26 \\
\hline $11 \mathrm{izt}$ & $37.34(2.41)$ & $18.82(1.51)$ & WHT+ISIS & 2011 Aug. 31 \\
\hline 11qax & $83.47(0.40)$ & $12.22(0.65)$ & KPNO4m+RC Spec & 2012 Jan. 26 \\
\hline 12 bro & $44.18(0.37)$ & $9.96(0.99)$ & P200+DBSP & 2012 Apr. 29 \\
\hline $12 \mathrm{bvh}$ & $89.80(0.95)$ & $30.76(0.46)$ & Lick 3-m+Kast & 2012 Jun. 14 \\
\hline $12 \operatorname{cod}^{*}$ & $57.91(1.88)$ & $3.76(0.22)$ & TNG+DOLORES & 2012 May 31 \\
\hline 12 fip & $38.47(0.94)$ & $9.62(0.93)$ & P200+DBSP & 2012 Jul. 21 \\
\hline $12 \mathrm{fo}$ & $116.10(0.96)$ & $19.53(0.66)$ & Keck1+LRIS & 2012 Apr. 29 \\
\hline $12 \mathrm{ftc}$ & $41.60(0.90)$ & $9.69(0.93)$ & P200+DBSP & 2012 Jul. 27 \\
\hline $12 \mathrm{gnn}^{*}$ & $42.30(0.94)$ & $19.29(1.59)$ & WHT+ISIS & 2012 Aug. 21 \\
\hline $12 \mathrm{hsx}$ & $46.21(0.25)$ & $15.77(0.90)$ & WHT+ISIS & 2012 Aug. 21 \\
\hline $13 \mathrm{aaz}$ & $74.24(1.42)$ & $32.27(0.83)$ & P200+DBSP & 2013 Jun. 03 \\
\hline $13 \mathrm{akg}$ & $58.19(2.42)$ & $19.82(0.54)$ & Keck2+DEIMOS & 2013 Jun. 06 \\
\hline $13 b j x$ & $62.72(0.47)$ & $22.82(0.62)$ & P200+DBSP & 2013 Aug. 03 \\
\hline 13bld & $33.95(0.45)$ & $27.58(2.27)$ & P200+DBSP & 2013 Jul. 05 \\
\hline $13 \mathrm{cnk}$ & $56.82(0.44)$ & $19.71(0.50)$ & Keck $2+$ DEIMOS & 2013 Oct. 04 \\
\hline $13 \mathrm{dkk}$ & $74.48(0.35)$ & $12.54(2.39)$ & P200+DBSP & 2013 Nov. 26 \\
\hline $13 \mathrm{dkz}$ & $49.73(0.44)$ & $14.73(0.80)$ & P200+DBSP & 2013 Nov. 02 \\
\hline $13 \mathrm{dla}^{*}$ & $75.52(0.43)$ & $16.50(5.79)$ & Keck1+LRIS & 2013 Dec. 02 \\
\hline $13 \mathrm{dqy}$ & $91.12(0.44)$ & $11.04(0.47)$ & P200+DBSP & 2014 Jan. 06 \\
\hline $14 \mathrm{adz}^{*}$ & $64.94(0.33)$ & $14.75(0.87)$ & Keck1+LRIS & 2014 May 28 \\
\hline 14aoi & $68.05(0.05)$ & $40.46(4.30)$ & Lick 3-m+Kast & 2014 Jun. 30 \\
\hline
\end{tabular}

Notes. (a) From explosion date, and corrected for time dilation. ${ }^{(*)}$ SN IIL. The other objects are SNe IIP. 
A\&A 587, L7 (2016)

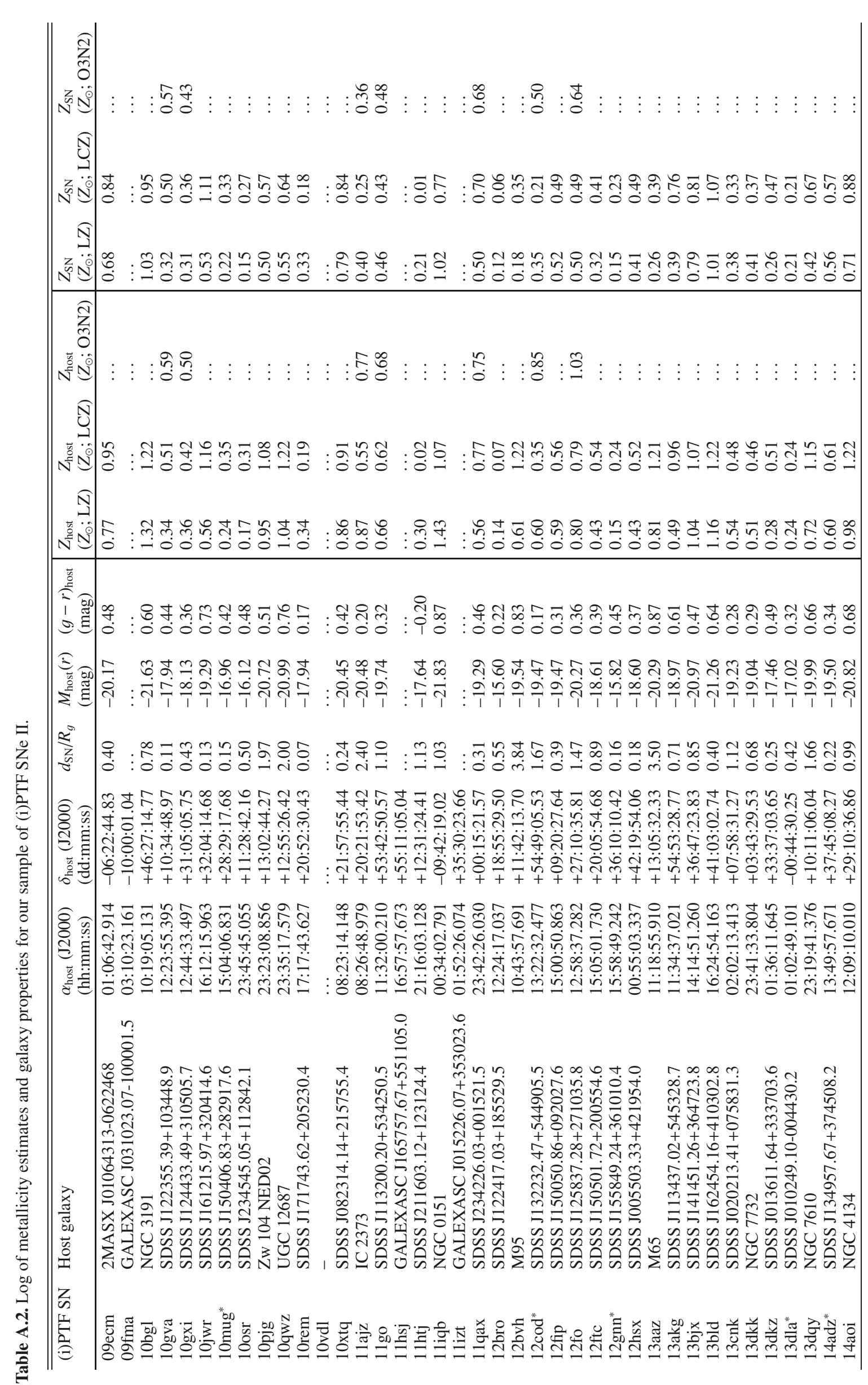

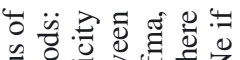

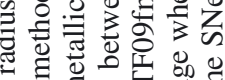

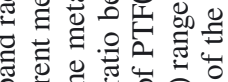
S.

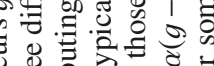
현 范

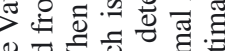

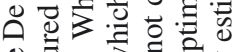

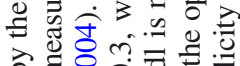

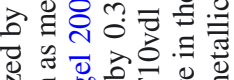

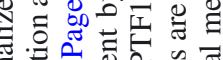
:

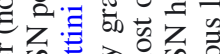

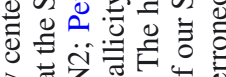

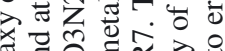

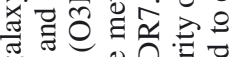

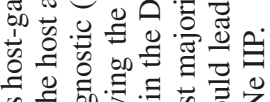

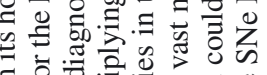

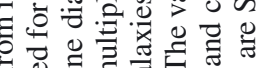

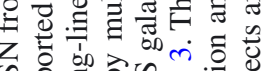
告

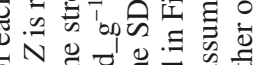
낭

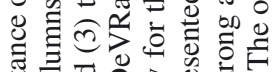
噌 च.

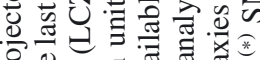

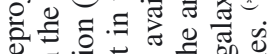

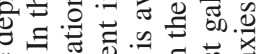

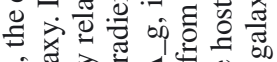

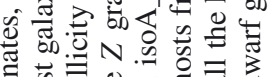

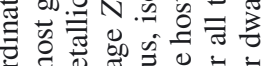

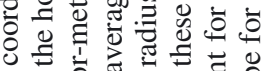

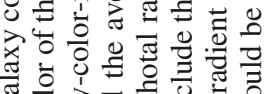
品 个.

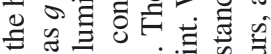
क न

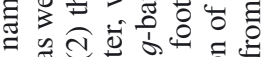

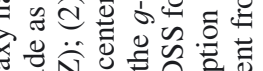

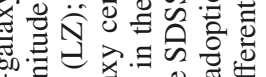

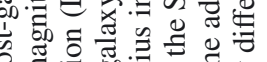

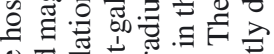
물

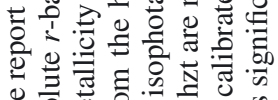

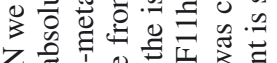

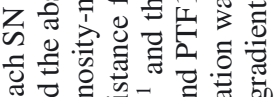
एँ

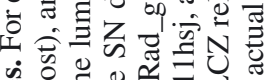

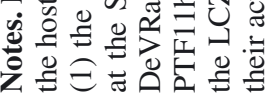

